\title{
Complete Response to Afatinib of an EGFR Exon 18 delE709_T710insD- Mutated Stage IV Lung Adenocarcinoma
}

\author{
Blandine Jelli ${ }^{1}$, Olivier Taton ${ }^{1}$, Nicky D'Haene ${ }^{2}$, Myriam Remmelink $^{2}$, Zita Mekinda $^{1}$ \\ ${ }^{1}$ Department of Pulmonology, Hôpital Erasme, Brussels, Belgium \\ ${ }^{2}$ Department of Pathology, Hôpital Erasme, Brussels, Belgium
}

Received: 26/06/2021

Accepted: 05/07/2021

Published: $11 / 08 / 2021$

How to cite this article: Jelli B, Taton O, D'Haene N, Remmelink Myriam, Mekinda Z. Complete response to afatinib of an EGFR exon 18 delE709_T710insDmutated stage IV lung carcinoma. EJCRIM 2021;8: doi:10.12890/2021_002749.

Conflicts of Interests: The authors declare there are no competing interests.

This article is licensed under a Commons Attribution Non-Commercial 4.0 License

\section{ABSTRACT}

Introduction: Epidermal growth factor receptor (EGFR) mutations are frequently found in patients with lung adenocarcinomas, $90 \%$ being deletions in exon 19 or point mutation in exon 21. Three generations of tyrosine kinase inhibitors (TKIs) targeting EGFR mutations are available and have changed patient prognosis but less data is available on exon 18 mutations and their sensitivity to TKI therapy. Exon 18 delE709_T710insD accounts for $0.06 \%(16 / 27,294)$ of all EGFR mutations and is an oncogenic driver. Several partial responses to afatinib have been described.

Case description: We report the first case, to the best of our knowledge, of the complete response to afatinib of a 57-year-old patient with stage IV lung adenocarcinoma with a deIE709_T710insD mutation in the EGFR gene detected by next-generation sequencing. Oral afatinib was prescribed and despite treatment interruptions and dosage tapering due to cutaneous adverse events, a complete response was achieved 12 months after treatment initiation and is currently maintained at 17 months.

Conclusion: When EGFR mutation is suspected, complete DNA sequencing of exons 18 to 21 should be carried out and we suggest that afatinib should be the first-line treatment for exon 18 delE709_T710insD-mutated advanced lung adenocarcinomas.

\section{LEARNING POINTS}

- Rare EGFR gene mutations are not detected by standard diagnostic kits.

- DNA sequencing is required to diagnose rare mutations of the EGFR gene.

- delE709_T710insD-mutated stage IV lung adenocarcinomas respond to afatinib.

\section{KEYWORDS}

EGFR mutation, exon 18, delE709_T710insD, lung cancer, afatinib

\section{INTRODUCTION}

Non-small cell lung cancers (NSCLC) represent 80 to $85 \%$ of all lung cancers with most patients diagnosed at an advanced or metastatic disease ${ }^{[1]}$. Fortunately, molecular drivers for oncogene addiction have been discovered over recent years (epidermal growth factor receptor (EGFR) mutations, rearrangements of the anaplastic lymphoma kinase (ALK) and c-ros oncogene 1 (ROS1) genes) that represent therapeutic targets and have tremendously changed patient prognosis.

EGFR mutations are found in $10-20 \%$ of Caucasian and up to $63 \%$ of Asian patients with lung adenocarcinomas ${ }^{[1,2]}$, especially in neversmokers and females. Those mutations occur most frequently in the 18 to 21 exons, $90 \%$ of these being deletions in exon 19 (Del19) or 
L858R substitution in exon 21, which confer sensitivity of the tumour to EGFR tyrosine kinase inhibitor (TKI) therapy. Less data is available on rare mutations (i.e., those in exon 18) making their sensitivity to TKI therapy less clear. EGFR-TKIs are now the standard of care as firstline treatment for stage III or IV NSCLC harbouring an activating EGFR mutation. Therefore, international guidelines recommend EGFR mutation testing before treating those patients.

We report here the first case of the complete response to afatinib of a patient with stage IV lung adenocarcinoma with a rare mutation in the EGFR gene.

\section{CASE DESCRIPTION}

A 57-year-old non-smoker north-African woman was admitted to the hospital with complaints of a 1-month shortness of breath and dry cough that appeared 2 years previously. She had no other systemic symptoms. Her past medical and surgical histories included a class I obesity, a reflux oesophagitis and an abortion by dilation and curettage. Vesicular murmur was diminished at the right lung base. The rest of the medical examination was normal. Standard blood tests were normal. A chest x-ray revealed a right pleural effusion. Thoracocentesis showed a lymphocytic exudative liquid. A chest CT scan confirmed a $7 \mathrm{~cm}$ mass of the right lower lobe responsible for a bronchus intermedius stenosis and associated with a pleural effusion. A bronchoscopy was performed with endobronchial biopsies of a mass in the bronchus intermedius. Pathologic examination showed lung parenchyma with adenocarcinoma positive for TTF1 but negative for ALK and ROS1 proteins. The PD-L1 Tumor Proportion Score (TPS) was evaluated at 30\%. DNA was extracted from the formalin-fixed paraffinembedded cell block and subjected to gene panel analysis using next-generation sequencing (NGS). Gene mutations were analyzed using the Colon and Lung Cancer Panel and Oncomine Solid Tumor-Plus Panel (AmpliSeq; Life Technologies, Merelbeke, Belgium) for sequencing hotspot mutations in 25 genes: AKT1, ALK, BRAF, CTNNB1, DDR2, EGFR, ERBB2, ERBB4, FBXW7, FGFR1, FGFR2, FGFR3, HRAS, KIT, KRAS, MAP2K1, MET, NOTCH1, NRAS, PDGFRA, PIK3CA, PTEN, SMAD4, STK11 and TP53. An atypical deletion in exon 18 of the EGFR gene was detected: p.E709_710>D with an allelic frequency of 48\% without any other molecular alteration. An 18FDG-positron emission tomography (PET) scan showed an increase in fluorodeoxyglucose (FDG) uptake in the right lower lobe mass (Fig. 1) and in several right pleural nodules. Brain magnetic resonance imaging (MRI) showed no cerebral metastasis. The final diagnosis was a cT4N1M1a (stage IV) lung adenocarcinoma according to the International Association for the Study of Lung Cancer (IASLC) and the American Joint Committee on Cancer (AJCC) 8th TNM classification.
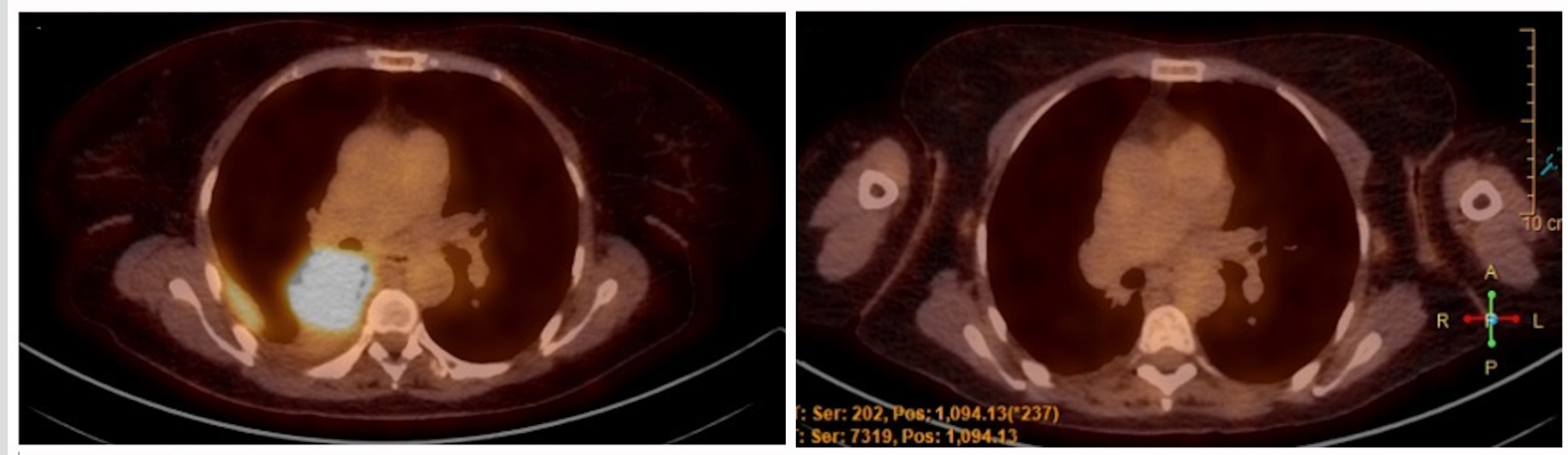

Figure 1. Evolution of the 18FDG-PET scan of the patient. Left: Initial 18FDG-PET scan of the patient. Right: 18 FDG-PET scan 14 months after the start of afatinib therapy

\section{DISCUSSION}

We describe the first case of a complete response to afatinib of a patient with stage IV lung adenocarcinoma with exon 18 delE709_T710insD mutation in the EGFR gene. Afatinib was chosen as the first-line treatment based on the partial response described in existing case reports ${ }^{[3,4]}$ in vitro studies ${ }^{[3,5]}$ and according to the pharmaceutical reimbursement criteria of TKIs. Despite moderate cutaneous adverse effects leading to a progressive tapering of the dose and several treatment interruptions, response to afatinib treatment was maintained. At 12 months, a complete response according to RECIST was obtained and the patient is currently pursuing the treatment.

As shown in Table 1, 12 cases of treated delE709_T710insD-mutated advanced lung adenocarcinomas have been described: two-thirds of the patients were Asian, 7 patients were female and half of them (including our patient) were treated with afatinib. 


\begin{tabular}{|c|c|c|c|c|c|c|c|c|c|c|c|}
\hline Patient & Sex & Age & Smoking & $\begin{array}{l}\text { Initial } \\
\text { staging }\end{array}$ & $\begin{array}{c}\text { Detection } \\
\text { method }\end{array}$ & $\begin{array}{l}\text { Treatment } \\
\text { line }\end{array}$ & TKI & $\begin{array}{l}\text { Dosage } \\
\text { (mg daily) }\end{array}$ & Response & PFS (m) & $O S(m)$ \\
\hline 1 [6] & $\mathrm{F}$ & 61 & no & IV & DS & N/A & Gefitinib & 250 & SD & 5.1 & 79.0 \\
\hline 2 [6] & M & 65 & yes & IV & DS & N/A & Gefitinib & 250 & PD & 0.9 & 11.1 \\
\hline $3[6]$ & $\mathrm{F}$ & 57 & no & IV & DS & N/A & Gefitinib & N/A & PD & 0.6 & 24.1 \\
\hline $4[6]$ & M & 79 & yes & IV & DS & N/A & Gefitinib & N/A & SD & 6.2 & 6.2 \\
\hline $5[6]$ & M & 68 & yes & IV & DS & $\mathrm{N} / \mathrm{A}$ & Gefitinib & N/A & PD & 2.3 & 29.5 \\
\hline $6[7]^{*}$ & $\mathrm{~F}$ & 88 & no & IV & DS & 1st & Erlotinib & $150=>75$ & PR & 6.0 & N/A \\
\hline $7[3]^{* *}$ & M & 63 & N/A & IV & DS & $\begin{array}{l}3 \text { rd } \\
\text { 4th }\end{array}$ & $\begin{array}{l}\text { Erlotinib } \\
\text { Afatinib }\end{array}$ & 15040 & $\begin{array}{l}\text { SD } \\
\text { PR }\end{array}$ & $\begin{array}{l}\text { N/A } \\
\text { N/A }\end{array}$ & N/A \\
\hline $8[4]$ & $\mathrm{F}$ & 52 & no & IV & DS & 1st & Afatinib & 40 & PR & N/A & N/A \\
\hline 9 [8] & $\mathrm{F}$ & 56 & no & IV & NGS & 6th & Afatinib & N/A & $P R$ & 7.0 & N/A \\
\hline $10[9]^{* * *}$ & $M$ & 71 & yes & IIIA & DS & $3 r d$ & Afatinib & 40 & PR & 11.0 & $>21.0$ \\
\hline 11 [10] & $\mathrm{F}$ & 57 & no & IIIB & NGS & 2 nd & Afatinib & 40 & PR & 12.0 & 36.0 \\
\hline $\begin{array}{l}12 \\
\text { (this case) }\end{array}$ & $\mathrm{F}$ & 57 & no & IV & NGS & 1st & Afatinib & $50=>20$ & $C R$ & $\begin{array}{l}17.0 \\
\text { (ongoing) }\end{array}$ & $\begin{array}{l}17.0 \\
\text { (ongoing) }\end{array}$ \\
\hline
\end{tabular}

Table 1. Summary of patients harbouring exon 18 delE709_T710insD-mutated lung adenocarcinoma treated with EGFR-TKIs. CR: complete response; DS: direct sequencing assay; EGFR: epidermal growth factor receptor; F: female; M: male; N/A: not available; NGS: next-generation sequencing assay; OS: overall survival; PD: progressive disease; PFS: progressionfree survival; PR: partial response; SD: stable disease; TKI: tyrosine kinase inhibitor.

${ }^{*}$ Clinical response was maintained for 6 months, but further erlotinib treatment was refused by patient. **Patient started afatinib therapy following erlotinib cutaneous adverse event. ${ }^{* *}$ Afatinib was combined with monthly $400 \mathrm{mg}$ intravenous bevacizumab. Adapted from I wamoto et al., 2019

The best response until now according to the RECIST criteria was a partial response with progression-free survival (PFS) of 12 months, supporting 2015 in vitro studies on the efficacy of afatinib on exon 18 mutations by Hirano et al. ${ }^{[5]}$ and Kobayashi et al. ${ }^{[3]}$.

Exon 18 mutations constitute $3.6 \%$ of all EGFR mutations and G719X substitutions account for most of these ${ }^{[3]}$. Substitutions or deletions in the E709 portion of exon 18 are less reported, deletion delE709_T710insD being extremely rare. According to the Catalogue of Somatic 
Mutations in Cancer (COSMIC) v94 database, this specific exon 18 deletion accounts for 0.06\% (16/27,294) of all EGFR mutations. delE709_ T710insD is an oncogenic driver ${ }^{[3]}$. Consequently, detecting this type of mutation is important for choosing an effective treatment but also because driver mutations are generally mutually exclusive ${ }^{[3]}$, allowing us to gain treatment time by skipping the search for another oncogenic driver and to propose adequate therapy. This highlights the importance of complete DNA sequencing of exons 18 to 21 , as some are not detected by many standard EGFR mutation testing kits.

This case strengthens other case reports and in vitro studies on the efficacy of afatinib on exon 18 delE709_T710insD-mutated advanced lung adenocarcinomas and we suggest that afatinib should be the first-line treatment in this particular diagnosis. It also shows the importance of detecting potential driver mutations with efficient diagnostic assays.

\section{REFERENCES}

1. Shi Y, Au JS, Thongprasert S, Srinivasan S, Tsai CM, Khoa MT, et al. A prospective, molecular epidemiology study of EGFR mutations in Asian patients with advanced non-smallcell lung cancer of adenocarcinoma histology (PIONEER). J Thorac Oncol 2014;9:154-162.

2. Harrison P, Vyse S, Huang P. Rare epidermal growth factor receptor (EGFR) mutations in non-small cell lung cancer. Semin Cancer Biol 2020;61:167-179.

3. Kobayashi Y, Togashi Y, Yatabe Y, Mizuuchi H, Jangchul P, Kondo C, et al. EGFR exon 18 mutations in lung cancer: molecular predictors of augmented sensitivity to afatinib or neratinib as compared with first- or third-generation TKIs. Clin Cancer Res 2015;21:5305-5313.

4. Ibrahim U, Saqib A, Atallah JP. EGFR exon 18 delE709_T710insD mutated stage IV lung adenocarcinoma with response to afatinib. Lung Cancer 2017;108:45-47.

5. Hirano T, Yasuda H, Tani T, Hamamoto J, Oashi A, Ishioka K, et al. In vitro modeling to determine mutation specificity of EGFR tyrosine kinase inhibitors against clinically relevant EGFR mutants in non-small-cell lung cancer. Oncotarget 2015;6:38789-38803.

6. Wu JY, Shih JY. Effectiveness of tyrosine kinase inhibitors on uncommon E709X epidermal growth factor receptor mutations in non-small-cell lung cancer. Onco Targets Ther 2016;11:6137-6145.

7. Ackerman A, Goldstein MA, Kobayashi S, Costa DB. EGFR delE709_T710insD: a rare but potentially EGFR inhibitor responsive mutation in non-small-cell lung cancer. J Thorac Oncol 2012;7:e19-e20.

8. Iwamoto Y, Ichihara E, Hara N, Nakasuka T, Ando C, Umeno T, et al. Efficacy of afatinib treatment for lung adenocarcinoma harboring exon 18 delE709_T710insD mutation. Jpn J Clin Oncol 2019;49:786-788.

9. An N, Wang H, Zhu H, Yan W, Jing W, Kong L, et al. Great efficacy of afatinib on a patient with lung adenocarcinoma harboring uncommon EGFR delE709_T710insD mutations: a case report. Onco Targets Ther 2019;12:7399-7404.

10. D'Haene N, Le Mercier M, Salmon I, Mekinda Z, Remmelink M, Berghmans T. SMAD4 mutation in small cell transformation of epidermal growth factor receptor mutated lung adenocarcinoma. Oncologist 2019;24:9-13. 